\title{
Alimentación en zona de operaciones y riesgo cardiovascular
}

La sociedad actual muestra un gran interés por la alimentación, dada la estrecha relación entre nutrición y salud. Desgraciadamente, este interés motivado por el deseo de disfrutar de una vida larga y sana, se acompaña de la proliferación de toda clase de recomendaciones dietéticas basadas en mitos y creencias irracionales, olvidando los principios científicos de la nutrición. Desde tiempos remotos, el hombre ha atribuido a algunos alimentos las virtudes más extraordinarias, que sorprendentemente aparecen más o menos disfrazadas en extravagantes dietas que circulan en la actualidad. La credulidad del hombre en materia de alimentación es ilimitada. De ahí la contínua aparición de regímenes alimenticios destinados a curar todo tipo de dolencias que encuentran terreno abonado en aquellas personas con un pensamiento mágico de la vida, en vez de científico, y que poco tiene que ver con el nivel cultural o educativo.

El objetivo de la alimentación en zona de operaciones es proporcionar una suficiente energía, ingesta proteica, vitamínica, sales minerales y tolerancia intestinal para mantener al combatiente en las mejores condiciones físicas y mentales. Oficiales, suboficiales, tropa y marinería destacados en zona de operaciones (Afganistán, Líbano, Índico, etc) presentan, no infrecuentemente diabetes, hipertensión arterial, hipercolesterolemia, obesidad y hábitos como el tabaquismo, que hacen que presenten un mayor riesgo cardiovascular.

Existe cierta preocupación acerca de la salud cardiovascular de las tropas destacadas en zonas de conflicto. En un artículo publicado en la Revista Ejército ${ }^{1}$ se ha sugerido la necesidad de disponer de raciones de combate con un menú vegetariano, como dieta capaz de disminuir la incidencia de enfermedades cardiovasculares, de determinados tipos de cáncer y que confiere una mayor longevidad. También, en ocasiones, ha sido solicitado al Servicio de Endocrinología y Nutrición del Hospital Central de la Defensa el diseño y facilitación de dietas para patologías específicas (hipercolesterolemia, diabetes, etc) con objeto de que aquellos militares que son enviados a zona de operaciones y que tienen un mayor riesgo cardiovascular, puedan seguir una alimentación más saludable.

La dieta vegetariana es un ejemplo de la confusión que se crea cuando se mezclan conceptos que pertenecen específicamente al campo de la nutrición, con ideas pseudofilosóficas desprovistas de significado desde el punto de vista de las necesidades nutricionales reales del organismo. El origen del vegetarianismo se atribuye al budismo y religiones primitivas de la India, relacionado con la prohibición de sacrificar animales, pero esta prohibición no se debe originariamente a un sentimiento de bondad y compasión hacia los animales, sino que se fundamenta en la doctrina de la transmigración de las almas. Esta creencia, que tiene su raíz en el mito de la inmortalidad sostiene que cuando una persona muere, si ha llevado una vida virtuosa, se reencarna en un nivel superior, mientras que si su conducta ha sido mala, lo hace en un nivel inferior. De esta forma, el más humilde insecto puede estar habitado por un alma humana.

La propuesta de incluir raciones de combate vegetarianas, se basa en el Adventist Health Study ${ }^{2}$. En este estudio fueron incluidos 34.192 hombres y mujeres, adventistas del séptimo día. La mayoría no fumaban ni bebían alcohol. Aproximadamente el 50\% eran vege- tarianos (nunca comían carne o lo hacían menos de una vez al mes) o semivegetarianos (comían carne menos de una vez por semana), el resto eran no vegetarianos y pocos eran veganos. El estudio duró 12 años, con una media de seguimiento de 6 años, el resultado fue de una mayor esperanza de vida entre los adventistas vegetarianos que entre los no vegetarianos, tanto en hombres como en mujeres, pero no se consiguió identificar que factores habían contribuido a la mayor longevidad. Análisis multivariante mostraron una asociación entre el consumo de carne de vaca ( $>3$ veces por semana) y la mortalidad por cardiopatía isquémica en comparación con los no vegetarianos. El riesgo de desarrollar cardiopatía isquémica fue un $37 \%$ menor en varones vegetarianos, la incidencia de carcinoma de colon y próstata fue mayor en no vegetarianos y los consumidores de carne de vaca tuvieron un mayor riesgo de carcinoma de vejiga. El alto consumo de frutas o frutos secos se asoció con un menor riesgo de carcinoma de pulmón, próstata y páncreas. Los adventistas del séptimo día vegetarianos tuvieron un menor riesgo de diabetes, hipertensión y artritis que los no vegetarianos. El alimento que con más consistencia se correlacionó con un menor riesgo de cardiopatía isquémica fatal y no fatal, fueron las nueces y en menor medida el pan integral. Un importante predictor de mortalidad por enfermedad coronaria en varones fue la edad a la que se hacían vegetarianos.

En conclusión, el estudio mostró que los vegetarianos tenían un menor riesgo de obesidad, hipertensión, diabetes, artritis, cáncer de colón y próstata, mortalidad por enfermedad coronaria y mortalidad global. El consumo de nueces y pan integral fueron factores protectores contra la enfermedad coronaria mientras que el consumo de carne de vaca aumentó el riesgo en varones, no así en mujeres. El consumo de legumbres y frutas ( $\geq 3$ veces/semana), pareció ser un factor protector contra diversos cánceres mientras que la carne de vaca probablemente aumentó el riesgo de cáncer de colon y vejiga. Es importante resaltar que los vegetarianos pudieron tener un riesgo menor por no consumir carne de vaca, pero es igualmente posible que esta protección pudiera ser debida al consumo de frutas verduras o nueces, siendo estas últimas las probablemente responsables ${ }^{3}$.

Ha sido demostrado que la dieta vegetariana no mejora el rendimiento físico en atletas y además, si no está bien planeada, puede ser carencial y por tanto empeorar el rendimiento físico ${ }^{4}$. En áreas de operaciones, en condiciones de frío o calor extremos y más en situaciones de combate, la forma física del soldado es fundamental, de forma que un bajo rendimiento físico puede poner en peligro su vida y la de los demás. No parece por tanto la dieta vegetariana la más adecuada para el combatiente. Por otro lado, fueron necesarios 12 años de estudio con un seguimiento medio de 6 años para demostrar las virtudes saludables de dicha dieta. No se ha demostrado que una dieta seguida tan sólo durante 6 meses, tenga ningún efecto sobre la salud cardiovascular, ni positivos ni negativos.

Tres tipos de dietas se han asociado con buena salud y longevidad, basado en evidencias geográficas, la dieta china, la japonesa y la mediterránea. El concepto de dieta mediterránea es originado a raíz del Seven Countries Study, iniciado por Keys en los años 50. El estudio mostraba que a pesar de una alta ingesta de grasas, la po- 
blación de la isla de Creta tenía una baja incidencia de enfermedad coronaria, ciertos tipos de cáncer y una larga esperanza de vida. Los patrones dietéticos tradicionales de Creta, gran parte de Grecia y Sur de Italia, en los años 60, fueron considerados responsables de la buena salud de estas regiones ${ }^{5}$.

Aunque no existe una única dieta mediterránea, puesto que 15 países bordean el Mar Mediterráneo, hay una serie de características comunes como son la abundancia de verduras, frutas, cereales integrales, nueces y legumbres, aceite de oliva como principal fuente de grasas, pescado, pollo consumido en cantidades moderadas, bajo consumo de carne roja y moderado consumo de vino con las comidas. Las dietas, mediterránea y asiáticas, comparten muchos elementos, como la alta ingesta de frutas, verduras, nueces, legumbres, cereales enteros y aceite de diferentes plantas. Sin embargo, los patrones dietéticos en el Mediterráneo están cambiando rápidamente con un aumento del consumo de grasas saturadas y carbohidratos refinados. Esto hace que la prevalencia de obesidad esté aumentando, debido a la occidentalización de la dieta, el aumento de la ingesta calórica y la disminución de la actividad física.

Aunque se ha dicho que la dieta mediterránea surgía de la pobreza alimentaria de la región, que obligaba al consumo principalmente de cereales, legumbres y verduras cocinadas con aceite de oliva, como principal fuente de calorías, es impensable que griegos y romanos llevaran a cabo tan importantes hazañas y creación de Imperios si no hubieran tenido una adecuada y nutritiva dieta. Podemos decir, que la base de la dieta mediterránea es el aceite de oliva, los cereales y el vino. Estos 3 elementos, constituyeron la mayor parte del comercio mediterráneo durante el Imperio Romano, llegando a fundar ciudades, como Vollubilis en Marruecos, cuya única función era el control del aceite de la región y facilitar su salida al mar.

El primer estudio que demuestra los beneficios para la salud de la dieta mediterránea fue el Lyon Diet Heart Study, en el cual, 605 pacientes que habían tenido un infarto de miocardio, fueron asignados a dieta de estilo mediterráneo o a una dieta que se parecía a la American Heart Association Step I diet. Los pacientes asignados a DM fueron estimulados para consumir más frutas, verduras y pescado y menos carnes rojas, y sustituir la mantequilla por margarinas enriquecidas en ácido linolénico para imitar el contenido en omega-3 de la dieta de Creta. Tras un seguimiento de 27 meses, la tasa de eventos coronarios se redujo en un $73 \%$ y la mortalidad en un $70 \%$, en el grupo de intervención ${ }^{6}$.

Más recientemente, un estudio con una dieta Indo-Mediterránea, en 1.000 pacientes en la India, con enfermedad coronaria o alto riesgo de enfermedad coronaria, el grupo de intervención en la dieta, caracterizado por aumento de la ingesta de mostaza, aceite de soja, nueces, verduras, frutas y cereales integrales, reducían la tasa de infarto de miocardio fatal en un $1 / 3$ y de muerte súbita de causa cardiaca en $2 / 3^{7}$.

Trichopoulus, en un estudio publicado en New England Journal of Medicine, estudia 22.043 adultos sanos en Grecia, y, para medir, el grado de seguimiento de la dieta, elabora un sistema de puntuación para la ingesta de verduras, frutas, nueces, legumbres, cereales, pescado, grasas monoinsaturadas, pollo, carne roja, productos lácteos y moderado consumo de alcohol. La media de seguimiento fue de 44 meses. El resultado fue que a mayor adherencia a la dieta mediterránea, la mortalidad cardiovascular y por cáncer fue menor, resultando significativa sólo en los $>55$ años, pero no en los menores de 55 años, quizá debido a un incremento acumulativo en el tiempo beneficioso de la dieta mediterránea o quizá por el bajo número de eventos que se produjeron en ese grupo de población. El beneficio fue mayor respecto a la mortalidad cardiovascular que por cáncer, aunque ambos resultaron significativos. Los únicos alimentos que resultaron predictivos de mortalidad fueron las frutas y nueces y el cociente ácidos grasos monoinsaturados/saturados. En este estudio, a pesar de la fuerte asociación entre la puntuación de la dieta mediterránea y la mortalidad, no se encontraron asociaciones entre los componentes individuales de la dieta. Una posible explicación sería que el efecto de cada uno de los nutrientes es demasiado pequeño para ser detectado, pero el efecto acumulativo o sinérgico es sustancial ${ }^{8}$.

En conclusión, podemos decir que en la literatura, ninguna dieta seguida durante 6 meses, que es el tiempo máximo que los combatientes permanecen en zona de operaciones, ha demostrado un efecto beneficioso o perjudicial desde el punto de vista cardiovascular. Las dietas específicas, vegetariana, mediterránea o de prevención secundaria cardiovascular, han precisado un seguimiento de varios años para demostrar un efecto beneficioso. Y por otro lado, este efecto beneficioso cardiovascular está alejado del objetivo que se persigue con la alimentación para el combate. Además, pensando en el riesgo cardiovascular de las tropas, también se debería recomendar la abstención de fumar en operaciones, cuando el peligro real viene dado por la propia naturaleza de la misión.

\section{BIBLIOGRAFÍA}

1. F. J Miguel Martínez. Menú vegetariano para raciones individuales de combate. Revista Ejército N.829. Mayo 2010.

2. Fraser GE, Lindsted KD, Beeson L. Effect of risk factor values on lifetime risk of anda ge at first coronary event. The Adventist Health Study. Am J Epidemiol 1995;142:746-58.

3. Fraser, GE, Shavlik DJ. Ten Years of Life. It is a Matter of Choice? Arch Intern Med 2001;161:1645-52.

4. Nieman DC. Physical fitness and vegetarian diets: is there a relation? Am J Clin Nutr 1999;70(suppl):570S-575S.

5. Willet WC, Sacks F, Trichopoulus A, et al. Mediterranean diet pyramid: a cultural model for healthy eating. Am J Clin Nutr 1995;61:Suppl:1402S-1406S.

6. de Lorgeril M, Renaud S, Mamelle N, et al. Mediterranean alpha-linolenic acid-rich diet in secondary prevention of coronary heart disease. Lancet 1994;343.1454-1459.

7. Singh RB, Dubnov G, Niaz MA, et al. Effect of a Indo-Mediterranean diet on progression of coronary artery disease in high risk patients (Indo-Mediterranean Diet Heart Study): a randomised single-blind trial. Lancet 2002;369:1455-1461.

8. Trichopoulou A, Costacou T, Bamia C, Trichopoulos D. Adherence to a Mediterranean Diet and Survival in a Greek Population. N Engl J Med 2003;348:25992068.

Arturo Lisbona Gil Teniente coronel Médico

José Antonio Rosado Sierra Licenciado en Medicina Servicio de Endocrinología y Nutrición Hospital Central de la Defensa Gómez Ulla Madrid. España. 